\title{
Analgesic practices and predictors of use in South Africa-based runners
}

\author{
R Thorpe, ${ }^{1}$ BSc (Physiotherapy), MSc (Exercise and Sports Physiotherapy); M Blockman, ${ }^{2}$ BPharm, MB ChB, PG Dip Int Res Ethics, MMed; \\ T Burgess, ${ }^{1}$ BSc (Physiotherapy), MHSc (Bioethics), PhD (Exercise Science) \\ ${ }^{1}$ Division of Physiotherapy, Department of Health and Rehabilitation Sciences, Faculty of Health Sciences, University of Cape Town, South Africa \\ ${ }^{2}$ Division of Clinical Pharmacology, Department of Internal Medicine, Faculty of Health Sciences, University of Cape Town, South Africa
}

Corresponding author: $R$ Thorpe (rowanthorpe.physio@gmail.com)

\begin{abstract}
Background. The use of analgesics is prevalent in runners, with an associated potential for serious harm. More information is needed regarding runners' practices and the factors that may influence their use of analgesics, to identify potential sources of misuse or abuse. Objectives. To describe South Africa (SA)-based runners' practices regarding use of analgesics, focusing on the types used, sources of information, and factors predicting use.

Methods. This study had a descriptive, cross-sectional design. SA-based runners aged $\geq 18$ years, who had run at least one race during the year preceding the study, were included. Participants were recruited via social media and SA running clubs. They completed an online survey that included demographic information, training and competition history, and analgesic usage practices.

Results. Data from 332 participants (196 females, 136 males) were analysed. There was a high rate of analgesic use (64\%), with 17\% of users reporting concomitant use of more than one type of analgesic. The highest rate of analgesic use was after a run (80\%). Non-steroidal antiinflammatory drugs (NSAIDs) were the most frequently used analgesics before (71\%), during (50\%) and after a run (74\%). Importantly, NSAIDs and a combination NSAID-containing analgesic were the most common analgesics used concomitantly (19\%). Most participants (90\%) used over-the-counter analgesics, $41 \%$ of them receiving no input from any health professional. Sustaining a running-related injury increased the likelihood of analgesic use almost three-fold $(\operatorname{Exp}(B)=2.6 ; 95 \%$ confidence interval $1.59-2.41 ; p=0.0001)$.

Conclusions. A large percentage of runners in our study displayed unsafe practices regarding analgesic use during training and competition, predominantly for perceived injury management. Importantly, the lack of education and recommendations regarding analgesics from health professionals is very concerning, as there is a risk of potentially life-threatening analgesic-induced adverse effects, especially as a high percentage were using two NSAIDs concomitantly. Knowledge of these practices, gained through this study, could allow for the development and implementation of corrective strategies to promote education and safe practice of analgesic use in runners.
\end{abstract}

S Afr Med J 2021;111(4):321-326. https://doi.org/10.7196/SAMJ.2021.v111i4.14635

Endurance running is one of the most popular sports worldwide. ${ }^{[1,2]}$ Increased participation can result in a higher risk of running-related injuries (RRIs), with the potential to increase analgesic use. ${ }^{[1,3]}$ However, there is limited research investigating RRIs, or other factors, as possible predictors for analgesic use in runners.

Use and misuse of analgesics is prevalent and increasing in sporting populations, but this is not supported by adequate knowledge of the effects and adverse effects of these drugs. ${ }^{[4]}$ Non-steroidal antiinflammatory drugs (NSAIDs) are one of the most common classes of analgesics used globally in the general population, ${ }^{[5,6]}$ which may be attributed to high over-the-counter (OTC) accessibility. ${ }^{[7]}$ Their significant risks of gastrointestinal, renal and cardiac adverse effects argue for appropriate knowledge prior to use, particularly during endurance exercise. Despite these risks, the rationales provided by athletes for analgesic use include perceptions of improved performance, and prophylactic management of injuries. ${ }^{[8]}$ However, the lack of scientific evidence to support these proposed rationales for analgesic use $\mathrm{e}^{[6]}$ brings into question the sources of information that are influencing these athletes' choices.

The apparent lack of knowledge regarding the safe use of analgesics, combined with OTC availability, potentially without professional input, is a major cause for concern. ${ }^{[8,9]}$

\section{Objectives}

To determine and describe practices regarding the use of analgesics in South Africa (SA)-based runners, focusing on the types of analgesics used, sources of information regarding analgesic use, and factors predicting use.

\section{Methods}

Ethical approval for the study was obtained from the Faculty of Health Sciences Human Research Ethics Committee (HREC), University of Cape Town (ref. no. 093/2016).

This study had a descriptive cross-sectional design.

\section{Participants}

Participants were recruited via online advertisements distributed to all SA running clubs listed on the Nedbank Runners Guide website (http://www.runnersguide.co.za) and through personal social media pages (Facebook and Twitter - @RThorpePhysio). Participants were included if they lived in SA, had internet access, were $\geq 18$ years of age, considered running to be their main sport, and participated in at least one race, of indeterminate distance, per year. Participants were excluded if they did not provide informed consent or failed to complete the knowledge section of the survey. 


\section{Sample size determination}

Sample size was calculated based on data from previous studies that determined prevalence rates of NSAID use in athletes. ${ }^{[8,10]}$ Based on these studies, ${ }^{[8,10]}$ the prevalence of NSAID use in athletes was between $56 \%$ and $75 \%$. An expected prevalence of $60 \%$ was selected, and if the worst expected frequency was $50 \%$, with confidence intervals (CIs) of $95 \%$, the required sample size was 208 participants.

\section{Measurement instrumentation: Questionnaire}

A self-developed and validated questionnaire was used to investigate the participants' practices regarding use of analgesics in running, as well as their training, competition and injury history.

The questionnaire consisted of four sections designed to address the main objectives of the study: (i) personal and medical information; (ii) training and race history, assessing preparation for, and performance in, training and competition; (iii) analgesic use, assessing participants' running-related use of analgesics to determine whether practices regarding the use of analgesics were 'safe' or 'unsafe' (in the questionnaire, analgesics were listed by their trade names, but for the interpretation of the results these have been combined into their generic names); and (iv) knowledge of and attitudes towards analgesics.

The questionnaire was submitted to a panel of three experts to be assessed for content and construct validity. The panel consisted of a sports physician, an exercise physiologist and a clinical pharmacologist, who were selected based on their experience and reputation in the field of clinical pharmacology and endurance sport. The questionnaire was submitted to the panel following ethical approval, with a request for their assistance in validating the questionnaire. The panel were requested to comment on the clarity, coherence and applicability of the questionnaire. The feedback from the panel was reviewed by the investigators, and appropriate modifications were made to the questionnaire. The final questionnaire was returned to the experts for consensus and approval, and was approved by the HREC.

\section{Procedure}

The final questionnaire was uploaded to the online survey website SurveyMonkey (www.surveymonkey.com). Participants were required to complete the informed consent process before gaining access to the questionnaire, and all participants were informed that responses were anonymous and IP addresses were not recorded. The survey was open for 1 month and was re-advertised after 3 weeks to remind participants of the study and to encourage completion of the survey.

It was the intention of the researchers to translate the questionnaire into Afrikaans, isiXhosa and isiZulu. However, owing to the rapidity of responses, the study was closed to further enrolment earlier than predicted, before the translation of the questionnaire could be completed.

\section{Statistical analyses}

Statistical analyses were performed using SPSS software (SPSS Statistics for Windows, version 23.0; IBM Corp., USA). A ShapiroWilk test was used to determine whether the data were normally distributed. Frequency tables and Pearson's $\chi^{2}$ measures of association were used for categorical variables, and an independent $t$-test was used for numerical variables. Odds ratios (ORs) and 95\% CIs were calculated using VassarStats (http://www.vassarstats.net/odds2x2. $\mathrm{html}$ ) to determine associations between individual variables and analgesic use. Only variables that showed significant associations on multivariate testing were included in binary logistic regression models to determine whether any variables were predictive of analgesic use in runners. Regression model variables included the use of recovery methods and injury history.

\section{Results}

\section{Participants}

A total of 332 participants, 196 females (59\%) and 136 males (41\%), were included. This was significantly larger than the required sample, as there was an excellent response to the study, which resulted in data collection being completed $>3$ weeks ahead of schedule. There were no significant differences between male and female participants in age, level of education and employment history (Table 1).

\section{Training and competition history}

More than two-thirds of the participants $(n=222 ; 67 \%)$ had been running for $<10$ years, the majority running $<40 \mathrm{~km}$ a week ( $n=191$; $58 \%)$. Participants completed a mean (standard deviation) number of 3 (2) marathons and 2 (1) ultra-marathons during the year. More than half of the participants $(n=185 ; 56 \%)$ used some form of recovery method after training or racing.

\section{Injury history}

More than half of the participants $(n=173 ; 52 \%)$ reported an RRI within the 6 months prior to completing the questionnaire, with $>20 \%$ of these participants suffering more than one RRI. Injury severity was separated into two groups: injuries that were severe enough to affect training or competition, and those that were not. Of the participants who suffered an RRI, $67 \%$ suffered injuries that affected running.

\section{Running-related use of analgesics}

Running-related use of analgesics was high (64\%), with $17 \%$ of these participants using more than one analgesic concurrently.

Analgesics were predominantly used after a run $(n=170 ; 80 \%)$, followed by during a run $(n=119 ; 56 \%)$ and before a run $(n=75 ; 35 \%)$. Of participants who reported use of analgesics, $29 \%$ used them after running, $24 \%$ used them during and after a run, and $17 \%$ used them before, during and after running.

Participants predominantly accessed OTC analgesics $(n=190 ; 90 \%)$, $41 \%$ with no healthcare practitioner recommendation. Pharmacists were only responsible for $25 \%$ of OTC recommendations, with friends and fellow runners (19\%) the other main source of OTC recommendations.

NSAIDs were the most common class of analgesic used before ( $n=53 ; 71 \%)$, during $(n=60 ; 50 \%)$ and after $(n=126 ; 74 \%)$ a run, followed by combination analgesics and paracetamol (Table 2). Combination analgesics refers to analgesics that contain multiple medicines and include Myprodol (ibuprofen-paracetamol-codeine phosphate) and Spasmend (mephenesin-paracetamol). The analgesic most commonly used before and after a run was the NSAID diclofenac, while Myprodol, the NSAID (i.e. ibuprofen combined with paracetamol-codeine phosphate) combination analgesic, was the most popular during a run and was used by $39 \%$ of runners (Table 3 ). Diclofenac and a combination analgesic, predominantly the NSAID (ibuprofen-paracetamol-codeine phosphate) combination, was the pairing most commonly used by participants who made use of more than one analgesic concurrently (19\%). This was followed by the combination of diclofenac and paracetamol (16\%). Paracetamol and a combination analgesic were used concurrently by $5 \%$ of participants.

The reasons for running-related analgesic use are set out in Table 4. Almost half of the participants who used more than one analgesic concomitantly gave the reason that 'a single type of 
Table 1. Sociodemographic characteristics and medical history of participants $(N=332)$

\begin{tabular}{|c|c|c|c|c|c|}
\hline Characteristics & Male $(N=136)$ & Female $(N=196)$ & Total $(N=332)$ & $t$ & $p$-value \\
\hline Age (years), mean (SD) & $39(10)$ & $38(10)$ & $38(10)$ & 1.24 & 0.22 \\
\hline Level of education, $n$ (\%) & & & & 7.48 & 0.38 \\
\hline Grade 7 or less & $2(2)$ & 0 & $2(1)$ & & \\
\hline Completion of high school or equivalent (e.g. matric) & $22(16)$ & $30(15)$ & $52(16)$ & & \\
\hline Diploma & $32(24)$ & $40(20)$ & $72(22)$ & & \\
\hline Bachelor's degree & $29(21)$ & $47(24)$ & $76(23)$ & & \\
\hline Honours degree & $26(19)$ & $46(24)$ & $72(22)$ & & \\
\hline Master's degree & $17(13)$ & $25(13)$ & $42(13)$ & & \\
\hline $\mathrm{PhD}$ & $7(5)$ & $4(2)$ & $11(3)$ & & \\
\hline Other & $1(1)$ & $4(2)$ & $5(2)$ & & \\
\hline Employment history (employed), $n$ (\%) & & & & 1.62 & 0.45 \\
\hline Yes & $127(93)$ & $176(90)$ & $303(91)$ & & \\
\hline No & $8(6)$ & $19(10)$ & $27(8)$ & & \\
\hline I prefer not to answer & $1(1)$ & $1(1)$ & $2(1)$ & & \\
\hline Monthly income (ZAR), $n$ (\%) & & & & 37.79 & $0.001^{*}$ \\
\hline No income & $9(7)$ & $20(10)$ & $29(9)$ & & \\
\hline $1000-4999$ & 0 & $3(2)$ & $3(1)$ & & \\
\hline $5000-9999$ & $5(4)$ & $22(11)$ & $27(8)$ & & \\
\hline $10000-19999$ & $21(15)$ & $61(31)$ & $82(25)$ & & \\
\hline $20000-30000$ & $31(23)$ & $41(21)$ & $72(22)$ & & \\
\hline$>30000$ & $56(41)$ & $30(15)$ & $86(26)$ & & \\
\hline I prefer not to answer & $14(10)$ & $19(10)$ & $33(10)$ & & \\
\hline
\end{tabular}

Table 2. Classes of analgesics used in running

\begin{tabular}{llll}
\hline \multirow{2}{*}{ Class } & \multicolumn{3}{c}{ Time of use } \\
\cline { 2 - 4 } & Before a run $(\boldsymbol{N}=\mathbf{7 5}), \boldsymbol{n}(\%)$ & During a run $(\boldsymbol{N = 1 1 9 ) ,} \boldsymbol{n}(\mathbf{\%})$ & After a run $(\boldsymbol{N}=\mathbf{1 7 0}), \boldsymbol{n}(\%)$ \\
\hline NSAIDs & $53(71)$ & $60(50)$ & $126(74)$ \\
Combination analgesics & $30(40)$ & $55(46)$ & $90(53)$ \\
Paracetamol & $26(35)$ & $43(36)$ & $57(34)$ \\
Other & $9(12)$ & $9(8)$ & $12(7)$ \\
Muscle relaxant & $5(7)$ & $9(8)$ & $9(5)$ \\
Cortisone & $3(4)$ & $3(3)$ & $2(1)$ \\
Codeine & 0 & $3(3)$ & $7(4)$ \\
NSAIDs = non-steroidal anti-inflammatory drugs. & & &
\end{tabular}

analgesic was not effective in reducing joint or muscle pain' ( $n=18$; $49 \%$ ), while nearly a third did so 'to aid in the recovery of a significant running injury' $(n=11 ; 30 \%)$.

\section{Factors associated with running-related analgesic use}

The odds of using analgesics was significantly higher in participants who reported a previous RRI (OR 2.68; 95\% CI 1.69 - 4.26).

Multivariate analysis revealed no significant differences between any descriptive and sociodemographic factors in the running-related use of analgesics.

Regression analysis confirmed that having a previous RRI significantly predicted the use of analgesics by almost three-fold when controlling for other variables $(\operatorname{Exp}(B)=2.6 ; 95 \%$ CI 1.59 - 4.21; $p=0.0001$ )

\section{Discussion}

\section{Running-related use of analgesics}

We found that $64 \%$ of participants reported running-related use of analgesics. The prevalence in our study is greater than the $25-60 \%$ prevalence of analgesic use previously reported in running and sports such as soccer and triathlons. ${ }^{[5,10-12]}$ The concomitant use of more than one type of analgesic by $17 \%$ of participants in our study is comparable to recent research, where $10-32 \%$ of runners used more than one type of analgesic concurrently. ${ }^{[13]}$

Most participants in our study who used analgesics (90\%) used OTC formulations, and only $25 \%$ did so on the advice of a pharmacist. However, we did not explore what advice was received from the pharmacist. Friends, family members and other runners $(n=93 ; 49 \%)$ were common sources of information regarding analgesic use. A common reason cited for performance-enhancing drug use in athletes is known as the 'false consensus effect. ${ }^{\text {'[14] }}$ This effect results in an increase in drug use in athletes because they overestimate the prevalence of drug use in fellow athletes ${ }^{[14,15]}$ and may explain why family, friends and fellow athletes can significantly influence the athlete's attitudes and practice regarding drug use. ${ }^{[14]}$ Although we were unable to substantiate the effect of these influences on the use of analgesics, we feel that they may play a similar role in runners. 
Table 3. Types of analgesics used in running

\begin{tabular}{|c|c|c|c|}
\hline \multirow[b]{2}{*}{ Type } & \multicolumn{3}{|c|}{ Time of use } \\
\hline & $\begin{array}{l}\text { Before a run }(N=75), \\
n(\%)\end{array}$ & $\begin{array}{l}\text { During a run }(N=119) \text {, } \\
n(\%)\end{array}$ & $\begin{array}{l}\text { After a run }(N=170), \\
n(\%)\end{array}$ \\
\hline \multicolumn{4}{|l|}{ NSAIDs } \\
\hline Aspirin & $8(11)$ & $2(2)$ & $16(9)$ \\
\hline Diclofenac & $40(53)$ & $36(30)$ & $106(62)$ \\
\hline Ibuprofen & $15(20)$ & $21(4)$ & $44(26)$ \\
\hline Celecoxib & $2(3)$ & $3(3)$ & $10(6)$ \\
\hline Other ${ }^{*}$ & $5(7)$ & $9(8)$ & $17(10)$ \\
\hline \multicolumn{4}{|l|}{ Combination analgesics } \\
\hline Ibuprofen-paracetamol-codeine phospate (Myprodol) & $26(35)$ & $46(39)$ & $73(43)$ \\
\hline Mephensin-paracetamol (Spasmend) & $7(9)$ & $15(13)$ & $29(17)$ \\
\hline \multicolumn{4}{|l|}{ Paracetamol } \\
\hline Paracetamol & $21(28)$ & $30(25)$ & $51(30)$ \\
\hline Other ${ }^{*}$ & $6(8)$ & $14(12)$ & $9(5)$ \\
\hline \multicolumn{4}{|l|}{ Muscle relaxant } \\
\hline Diazepam & 0 & 0 & $1(1)$ \\
\hline Other ${ }^{*}$ & $5(7)$ & $9(8)$ & $10(6)$ \\
\hline \multicolumn{4}{|l|}{ Corticosteroids } \\
\hline Corticosteroids & $5(7)$ & $1(1)$ & $1(1)$ \\
\hline Other* & 0 & $2(2)$ & $1(1)$ \\
\hline \multicolumn{4}{|l|}{ Codeine } \\
\hline Codeine & 0 & $2(2)$ & $4(2)$ \\
\hline Other* & 0 & $1(1)$ & $4(2)$ \\
\hline Other analgesics & $9(12)$ & $9(8)$ & $12(7)$ \\
\hline
\end{tabular}

\section{Table 4. Reasons for analgesic use}

\begin{tabular}{ll}
\hline Reason & $\begin{array}{l}\text { Participants } \\
(\mathbf{N = 2 3 4 ) ,} \boldsymbol{n}(\mathbf{\%})\end{array}$ \\
\hline To alleviate headache & $138(59)$ \\
To reduce joint or muscle pain & $111(47)$ \\
Because of illness (flu, fever, nausea, etc.) & $94(40)$ \\
To aid recovery from a running injury & $76(33)$ \\
To decrease joint or muscle stiffness & $50(21)$ \\
To help with sleeping & $24(10)$ \\
To aid recovery after surgery & $14(6)$ \\
To reduce tiredness/fatigue & $12(5)$ \\
To prevent injury & $6(3)$ \\
Other & $12(5)$ \\
&
\end{tabular}

NSAIDs are regarded the first-line analgesic for the management of sports injuries. ${ }^{[5]}$ In our study, they were found to be the commonest class of analgesic used before, during and after a run. Diclofenac was the most commonly used before and after a run, with Myprodol, the NSAID combination analgesic, used the most during a run. The use of Myprodol during running is of major concern, as it is a combination containing the NSAID ibuprofen, as well as paracetamol and codeine. It should be noted that diclofenac and Myprodol was the most common pairing of analgesics in our study. Their use in combination presents a major risk for additive NSAID toxicity, as runners are unknowingly combining the ibuprofen-containing Myprodol with another NSAID.

Similarly, there may be an increased risk of unintentional paracetamol overdose, as both combination analgesics used in our study, Myprodol and Spasmend, contain paracetamol. Paracetamol is a commonly used analgesic ${ }^{[16,17]}$ and was the third most used class of analgesic in our study. It was paired with combination analgesics by $5 \%$ of participants who used more than one analgesic concurrently. This practice is as concerning as the combined use of Myprodol with another NSAID, and while the adverse effects and risks may be different from those associated with excessive NSAID dosing, they are no less serious.

Paracetamol is popular because of its perceived safety and low risk of systemic adverse effects in therapeutic and low doses, up to $4000 \mathrm{mg}$ per day. ${ }^{[16-19]}$ However, despite this perceived safety, paracetamol is a common cause of acute liver failure due to unintentional overdose, ${ }^{[17,18,20]}$ as it can result in hepatotoxicity, hepatic necrosis and nephrotoxicity, which can be fatal. ${ }^{[17,18,21]}$ In a multicentre US study in patients with an unintentional overdose, $63 \%$ were using acetaminophen (paracetamol)/opioid compounds. ${ }^{[22]}$

Toxicity is unusual from a single dose of paracetamol $<150 \mathrm{mg} / \mathrm{kg}$, or 7.5 - $10 \mathrm{~g}$ for an adult; however, it is likely to occur when $>250 \mathrm{mg} /$ $\mathrm{kg}$ body weight or $>12 \mathrm{~g}$ are consumed over a 24-hour period. ${ }^{[23-25]}$ We note that if full daily doses of monocomponent paracetamol, i.e. $4000 \mathrm{mg}$, were added to the regulated dosages of the combination analgesics, there is a risk that a runner may inadvertently consume $>7.5 \mathrm{~g}$ of paracetamol, and therefore be at risk of acute paracetamol toxicity.

\section{Factors associated with running-related analgesic use}

An RRI during the 6 months prior to the study predicted analgesic use in running, possibly because participants continued to run through injury to maintain fitness or to maintain participation. As a result, there is the potential for the injury to worsen as vulnerable 
tissues are overloaded with insufficient recovery time. There is also the risk that the use of analgesics, specifically NSAIDs, could delay the healing process, resulting in poor recovery and long-term injuries. ${ }^{[26]}$ Long-term NSAID use can increase the risk of disordered healing in bones and tendons up to two-fold. ${ }^{[26]}$ However, further research into these risks is required. ${ }^{[27]}$ Runners need to be aware of how RRIs may be exacerbated by analgesic use.

Our participants were more likely to use analgesics after than before or during a run, which we assume was to manage pain, stiffness or injuries from the run, and to allow for enhanced recovery. ${ }^{[28-30]}$ Most of our participants used analgesics to assist in the recovery of an RRI, despite there being no evidence to support their use in recovery. ${ }^{[31,32]}$ The high use of analgesics, especially NSAIDs during and after a run, is concerning, as there is an increased risk of dehydration at this stage. ${ }^{[33]}$ The use of NSAIDs in a dehydrated runner can result in acute renal failure. ${ }^{[34]}$ It is evident that increased awareness of the serious risks of analgesic use after a run and in a dehydrated state is needed.

Despite the various regulatory restrictions surrounding analgesics, the most commonly used combination analgesic in our study, Myprodol, is schedule 3, requiring a doctor's prescription. However, limited doses may be prescribed OTC under a pharmacist's supervision as schedule 2, as the regulations allow for pharmacist initiation under certain circumstances. ${ }^{[3,36]}$ These prescriptions and doses are recorded and closely monitored to prevent overpurchasing and misuse of these analgesics. The OTC accessibility could be limiting the interaction between health professionals and the consumer. ${ }^{[37,38]}$ It has been found that only small numbers of analgesic users are receiving professional medical recommendations regarding the appropriate use of analgesics. ${ }^{[39]}$ The resulting lack of consultation regarding the effects and adverse effects is an urgent concern that needs to be addressed.

\section{Recommendations}

The ways in which runners are educated regarding analgesic use should be further investigated to determine the best means to address the high use and potentially unsafe practices. The roles of social media, running clubs and various media sources should be explored.

As OTC use is high, the role of the pharmacist in the provision of education and advice must be evaluated further. This role is important, as OTC NSAIDs and combination analgesics can only be accessed in a pharmacy. It should be noted that the combination analgesic Myprodol can be obtained via prescription or pharmacist initiation only, providing a further opportunity for pharmacists' intervention.

\section{Study limitations}

We relied on self-reported data, which could not be independently verified. This may have biased the results due to recall.

The use of topical analgesics was not thoroughly investigated in our study. Topical analgesics, including NSAIDs, have been found to be as effective as some oral analgesics, with fewer adverse effects, and further investigation into topical analgesic use may be warranted. ${ }^{[40]}$

\section{Conclusions}

This study identified that there are large numbers of runners who are using analgesics during training and competition, and that in the majority of cases, practices were unsafe. A major concern is a lack of education and recommendations regarding analgesics from health professionals. Combined with the concomitant use of different analgesics, especially NSAIDs and paracetamol, this is very concerning, as it increases the risk of analgesic-induced adverse effects, which are potentially life-threatening.

It is essential for health professionals to provide improved education to runners regarding the safe use of analgesics, importantly the concomitant use of more than one NSAID. We believe that the scheduling and regulatory access of Myprodol in our country provides an important opportunity for healthcare professional intervention, which seems not to be occurring. Only via a concerted effort by runners, running club committees, competition organisers, social media and health professionals will the safe use of analgesics in running be inculcated in SA-based runners.

\section{Declaration. None.}

Acknowledgements. We thank Sean Beckett for his assistance with the statistical analyses. We also thank all the participants for the time that they volunteered to complete the survey.

Author contributions. TB and MB contributed to the study conception and design of the questionnaire and helped draft the manuscript. TB also assisted with statistical analyses. RT designed the questionnaire, performed statistical analyses and drafted the manuscript. All authors approved the final manuscript.

Funding. None.

Conflicts of interest. None.

1. Van Mechelen W. Running injuries. Sport Med 1992;14(5):320-335. https://doi.org/10.2165/00007256199214050-00004

2. Van Gent RN, Siem D, van Middelkoop M, van Os AG, Bierma-Zeinstra SMA, Koes BW. Incidence and determinants of lower extremity running injuries in long distance runners: A systematic review. Br J determinants of lower extremity running injuries in long distance runners:
Sports Med 2007;41(8):469-480. https://doi.org/10.1136/bjsm.2006.033548

3. Joslin JD, Lloyd JJB, Kotlyar T, Wojcik SMS. NSAID and other analgesic use by endurance runners 3. Joslin JD, Lloyd JJB, Kotlyar T, Wojcik SMS. NSAID and other analgesic use by endurance runners
during training, competition and recovery. S Afr J Sport Med 2013;25(4):101-104. https://doi. org/10.17159/2413-3108/2013/v25i4a340

4. Wynne HA, Long A. Patient awareness of the adverse effects of non-steroidal anti-inflammatory drugs (NSAIDs). Br J Clin Pharmacol 1996;42(2):253-256. https://doi.org/10.1046/j.1365-2125.1996.41420.x 5. Derman E, Schwellnus M. Pain management in sports medicine: Use and abuse of anti-inflammatory and other agents. S Afr Fam Pract 2014;52(1):27-32. https://doi.org/10.1080/20786204.2010.10873927 Warden SJ. Prophylactic use of NSAIDs by athletes: A risk/benefit assessment. Phys Sportsmed 2010;38(1):132-138. https://doi.org/10.3810/psm.2010.04.1770

Stosic R, Dunagan F, Palmer H, Fowler T, Adams I. Responsible self-medication: Perceived risks and benefits of over-the-counter analgesic use. Int J Pharm Pract 2011;19(4):236-245. https://doi. org/10.1111/j.2042-7174.2011.00097.x

8. Warner DC, Schnepf G, Barrett MS, Dian D, Swigonski NL. Prevalence, attitudes, and behaviors related to the use of nonsteroidal anti-inflammatory drugs (NSAIDs) in student athletes. J Adolesc Health 2002;30(3):150-153. https://doi.org/10.1016/s1054-139x(01)00325-1

9. Warden SI. Prophylactic misuse and recommended use of non-steroidal anti-inflammatory drugs by 9arden SJ. Prophylactic misuse and recommended use of non-steroidal anti-inflammat
athletes. Br J Sports Med 2009;43(8):548-549. https://doi.org/10.1136/bjsm.2008.056697

athletes. Br J Sports Med 2009;43(8):548-549. https://doi.org/10.1136/bjsm.2008.056697
10. Gorski T, Cadore EL, Pinto SS, et al. Use of NSAIDs in triathletes: Prevalence, level of awareness and . Gorski T, Cadore EL, Pinto SS, et al. Use of NSAIDs in triathletes: Prevalence, level of awaren
reasons for use. Br J Sports Med 2011;45(2):85-90. https://doi.org/10.1136/bjsm.2009.062166

11. Küster M, Renner B, Oppel P, Niederweis U, Brune K. Consumption of analgesics before a marathon and the incidence of cardiovascular, gastrointestinal and renal problems: A cohort study. BMJ Open 2013;3(4):e002090. https://doi.org/10.1136/bmjopen-2012-002090

12. Tscholl P, Junge A, Dvorak J. The use of medication and nutritional supplements during FIFA World Cups 2002 and 2006. Br J Sports Med 2008;42(9):725-730. https://doi.org/10.1136/bjsm.2007.045187

13. Locquet $M$, Beaudart $C$, Larbuisson $R$, et al. Self-administration of medicines and dietary supplements among female amateur runners: A cross-sectional analysis. Adv Ther 2017;33(12):2257-2268. https:// doi.org/10.1007/s12325-016-0426-2

14. Uvacsek M, Nepusz T, Naughton DP, Mazanov J, Ránky MZ, Petróczi A. Self-admitted behavior and perceived use of performance-enhancing vs psychoactive drugs among competitive athletes. Scand J
Med Sci Sports 2011:21(2):224-234. https://doi.org/10.1111/.1.1600-0838.2009.01041.x

15. Morente-Sánchez J, Zabala M. Doping in sport: A review of elite athletes' attitudes, beliefs, and . Morente-Sánchez J, Zabala M. Doping in sport: A review of elite athletes' attitudes,
knowledge. Sport Med 2013;43(6):395-411. https://doi.org/10.1007/s40279-013-0037-x

16. Bertolini A, Ferrari A, Ottani A, Guerzoni S, Tacchi R, Leone S. Paracetamol: New vistas of an old drug. CNS Drug Rev 2006;12(3-4):250-275. https:///doi.org/10.1111/j.1527-3458.2006.00250.x
CNS

17. James LP, Mayeux PR, Hinson JA. Acetaminophen-induced hepatotoxicity. Drug Metab Dispos 2003;31(12):1499-1506. https://doi.org/10.1124/dmd.31.12.1499

18. Mort JR, Shiyanbola OO, Ndehi LN, Xu Y, Stacy JN. Opioid-paracetamol prescription patterns and liver dysfunction: A retrospective cohort study in a population served by a US health benefit organization. Drug Saf 2011;34(11):1079-1088. https://doi.org/10.2165/11593100-000000000-00000

19. Gokhale M, Martin BC. Prescription-acquired acetaminophen use and potential overuse patterns 2001 - 2008. Pharmacoepidemiol Drug Saf 2012;21(2):226-230. https://doi.org/10.1002/pds.2235

20. Fosnocht D, Taylor JR, Caravati EM. Emergency department patient knowledge concerning acetaminophen (paracetamol) in over-the-counter and prescription analgesics. Emerg Med J 2008;25(4):213-216. https://doi.org/10.1136/emj.2007.053850

21. Kaufman DW, Kelly JP, Rohay JM, Malone MK, Weinstein RB, Shiffman S. Prevalence and correlates of exceeding the labeled maximum dose of acetaminophen among adults in a U.S. based internet survey. exceeding the labeled maximum dose of acetaminophen among adults in a U.S. based int
Pharmacoepidemiol Drug Saf 2012;21(12):1280-1288. https://doi.org/10.1002/pds.3350

22. Larson AM, Polson J, Fontana RJ, et al. Acetaminophen-induced acute liver failure: Results of a United States multicenter, prospective study. Hepatology 2005;42(6):1364-1372. https://doi.org/10.1002 hep. 20948 
23. Lewis RK, Paloucek FP. Assessment and treatment of acetaminophen overdose. Clin Pharm 1991;10(10):765-774

24. Prescott LF. Paracetamol overdosage: Pharmacological considerations and clinical management. Drugs 1983;25:290-314. https://doi.org/10.2165/00003495-198325030-00002

25. Makin AJ, Wendon J, Williams R. A 7-year experience of severe acetaminophen-induced hepatotoxicity (1987 - 1993). Gastroenterology 1995;109(6):1907-1916. https://doi.org/10.1016/0016hepatotoxicity (1987-

26. Lisowska B, Kosson D, Domaracka K. Positives and negatives of nonsteroidal anti-inflammatory drugs in bone healing: The effects of these drugs on bone repair. Drug Des Devel Ther 2018;12:1809-1814. https://doi.org/10.214//dddt.s164565

27. Marquez-Lara A, Hutchinson ID, Nuñez F, Smith TL, Miller AN. Nonsteroidal anti-inflammatory drugs and bone-healing. JBJS Rev 2016;4(3):e4. https://doi.org/10.2106/jbjs.rvw.0.0005

28. Taunton JE. A retrospective case-control analysis of 2002 running injuries. Br J Sports Med 2002;36(2):95-101. https://doi.org/10.1136/bjsm.36.2.95

29. Clough PJ, Shepherd J, Maughan RJ. Marathon finishers and pre-race drop-outs. Br J Sports Med 1989;23(2):97-101. https://doi.org/10.1136/bjsm.23.2.97

30. Satterthwaite P, Larmer P, Gardiner J, Norton R. Incidence of injuries and other health problems in the Auckland Citibank marathon, 1993. Br J Sports Med 1996;30(4):324-326. https://doi.org/10.1136 bjsm.30.4.324

31. Cheung K, Hume PA, Maxwell L. Delayed onset muscle soreness. Sport Med 2003;33(2):145-164. https://doi.org/10.2165/00007256-200333020-00005

32. Connolly DAJ, Sayers SE, McHugh MP. Treatment and prevention of delayed onset muscle soreness. J Strength Cond Res 2003;17(1):197-208. https://doi.org/10.1519/00124278-200302000-00030

33. Lipman G, Shea K, Christensen M, et al. Ibuprofen versus placebo effect on acute kidney injury in ultramarathons: A randomised controlled trial. Emerg Med J 2017;34(10):637-642. https://doi. org/10.1136/emermed-2016-206353
34. Ulinski T, Guigonis V, Dunan O, Bensman A. Acute renal failure after treatment with non-steroidal anti-inflammatory drugs. Eur J Pediatr 2004;163(3):148-150. https://doi.org/10.1007/s00431-003-

35. South African Government. Medicines and Related Substances Act (Act No. 101 of 1965). https://www. gov.za/documents/drugs-control-act-7-jul-1965-0000\# (accessed 21 February 2021).

36. South African Health Products Regulatory Authority. Public warning on the safe use of non-steroidal anti-inflammatory drugs (NSAIDs). 25 July 2018. http://196.15.225.13/index.php/2014-03-17-0948-36/2014-03-17-09-49-50? download=2855: media-statement-safe-use-of-non-steroidal-antiinflamatory-drugs\&start=45 (accessed 21 February 2021 .

37. Cham E, Hall L, Ernst A, Weiss S. Awareness and use of over-the-counter pain medications. South Med J 2002;95(5):529-535. https://doi.org/10.1097/00007611-200205000-00013

8. Albert S, Bix L, Bridgeman M, Carstensen L, et al. Promoting safe and effective use of OTC medications: CHPA-GSA National Summit. Gerontologist 2014;54(6):909-918. https://doi.org/10.1093/geront/ gnu034

39. Pottegård A, Kviesgaard A-K, Hesse U, Moreno SI, Hansen JM. Patient characteristics among users of analgesic over-the-counter aspirin in a Danish pharmacy setting. Int J Clin Pharm 2014:36(4):693-696. https://doi.org/10.1007/s11096-014-9968-z

40. Argoff CE. Targeted topical peripheral analgesics in the management of pain. Curr Pain Headache Rep 2003; $7(1): 34-38$. https://doi.org/10.1007/s11916-003-0007-3

Accepted 3 December 2020 